\title{
Dry reaction of dialkyl phosphite with aldehydes in the presence of acetic anhydride for the synthesis of 1-acetoxyphosphonates on solid bases
}

\author{
Babak Kaboudin* and Masoumeh Karimi \\ Department of Chemistry, Institute for Advanced Studies in Basic Sciences (IASBS), Gava Zang, \\ Zanjan 45195-1159, Iran \\ E-mail: kaboudin@iasbs.ac.ir
}

\begin{abstract}
Reaction of benzaldehyde with diethyl phosphite and acetic anhydride successively on different solid bases (oxides and carbonates) without the use of solvent were investigated. A simple, efficient, and new method has been developed for the synthesis of $\alpha$-acetoxyphosphonates from aldehydes through a one-pot reaction of aldehydes with diethyl phosphite in the presence of acetic anhydride under solvent-free conditions using potassium or sodium carbonate. This method is easy, rapid, and high yielding for the one-pot synthesis of $\alpha$-acetoxyphosphonates from aldehydes.
\end{abstract}

Keywords: Phosphonic esters, aldehydes, addition reaction, acetic anhydride, potassium carbonate, solid bases

\section{Introduction}

Reactions under heterogeneous conditions are of growing interest, ${ }^{1}$ because of their advantages of ease of set up, mild conditions, rapid reactions, selectivity, increased yields of the products, and low cost compared with their homogeneous counterparts. Organophosphorus compounds have found wide application in the areas of industrial, agricultural, and medicinal chemistry owing to their biological and physical properties, as well as their utility as synthetic intermediates. ${ }^{2} \alpha$-Functionalized phosphonic acids are valuable intermediates for the preparation of medicinal compounds and synthetic intermediates. ${ }^{3}$ Among $\alpha$-functional phosphonic acids, $\alpha$ acetoxyphosphonates and $\alpha$-hydroxyphosphonates are important classes of compounds that exhibit a variety of interesting and useful properties. In recent years, the preparation of $\alpha$ acetoxy- and $\alpha$-hydroxyphosphonates has attracted significant attention, due to their potential biological activities with broad applications such as enzyme inhibitors or as dinucleotide analogues having antiviral properties. ${ }^{4}$ In addition, they are useful intermediates in the synthesis 
of other phosphorus compounds. ${ }^{5}$ These compounds may also be used as precursors for the synthesis of optically active $\alpha$-hydroxyphosphonates. ${ }^{6}$ Indeed, $\alpha$-acetoxyphosphonates are also used as precursors for the synthesis of a variety of $\alpha$-substituted phosphonates.

In contrast to the widely studied $\alpha$-hydroxyphosphonic acid derivatives, ${ }^{7}$ relatively few papers have reported on the chemistry of $\alpha$-acetoxyphosphonates. Many effective methods for the preparation of $\alpha$-hydroxyphosphonates have been developed, but, to the best of our knowledge, few synthetic routes to $\alpha$-acetoxyphosphonates have been reported. These methods involve prolonged heating of acyl phosphates with a carbonyl compound at $120{ }^{\circ} \mathrm{C},{ }^{8}$ direct acetylation of $\alpha$-hydroxyphosphonates with ketenes catalyzed by $\mathrm{BF}_{3}$.OEt ${ }^{9}$ or $\mathrm{H}_{2} \mathrm{SO}_{4},{ }^{10}$ and acetylation of $\alpha$-hydroxyphosphonates with $\mathrm{Ac}_{2} \mathrm{O}$ or $\mathrm{AcCl}$ in the presence of $\mathrm{Et}_{3} \mathrm{~N}$ or pyridine as base. ${ }^{11}$ Recently, a new method has been reported, using copper triflate as a catalyst for the preparation of $\alpha$-acetoxyphosphonates from reaction of $\alpha$-hydroxyphosphonates with $\mathrm{Ac}_{2} \mathrm{O}{ }^{12}$ However, these methods have problems, including harsh reaction conditions, low yields, long reaction times, using of Lewis acids, and side reactions. As part of our efforts to explore the utility of solid phase reactions for the synthesis of organophosphorus compounds, ${ }^{13}$ we report a new method for the one-pot synthesis of $\alpha$-acetoxyphosphonates from the reaction of diethyl phosphite with aldehydes in the presence of $\mathrm{Ac}_{2} \mathrm{O}$ under solvent-free conditions, using $\mathrm{K}_{2} \mathrm{CO}_{3}$ or $\mathrm{Na}_{2} \mathrm{CO}_{3}$ as a solid base, producing high yields of $\alpha$-acetoxyphosphonates.

\section{Results and Discussion}

The reaction of diethyl phosphite with benzaldehyde, as a model compound, in the presence of acetic anhydride under dry conditions was examined (Table 1). The results showed that the reaction yield and time seem to depend on the basicity of the solid.

The reaction with basic solids such as $\mathrm{MgO}, \mathrm{CaO}$ and $\mathrm{BaO}$ gave the desired product with

moderate to good yields. $\mathrm{MgO}$ gave a better yield than the other metal oxides. ${ }^{13 \mathrm{e}}$ The best result was obtained with an alkali- carbonate (potassium- or sodium-). With alumina and $\mathrm{SiO}_{2}$ the yield increased when the reaction was performed under microwave irradiation.

Then potassium carbonate was selected as a convenient solid base for the synthesis of 1acetoxyphosphonates. This process was successfully applied to other aldehydes (1), as summarized in Table 2. As shown in Scheme 2 and Table 2, the reaction of a mixture of diethyl phosphite and aromatic aldehydes $(\mathbf{1 a}-\mathbf{1 k})$ with $\mathrm{Ac}_{2} \mathrm{O}$ under solvent-free condition using $\mathrm{K}_{2} \mathrm{CO}_{3}$ afforded the desired products in high yields (2a-2k). Naphthalene carboxaldehydes as polynuclear aldehydes also reacted with diethyl phosphite in the presence of $\mathrm{Ac}_{2} \mathrm{O}$ using $\mathrm{K}_{2} \mathrm{CO}_{3}$, to give the desired compounds in high yields (2l and $\mathbf{2 m})$. Cinnamaldehyde (1n) also reacted to give the desired compound $\mathbf{2 n}$ in good yield. 


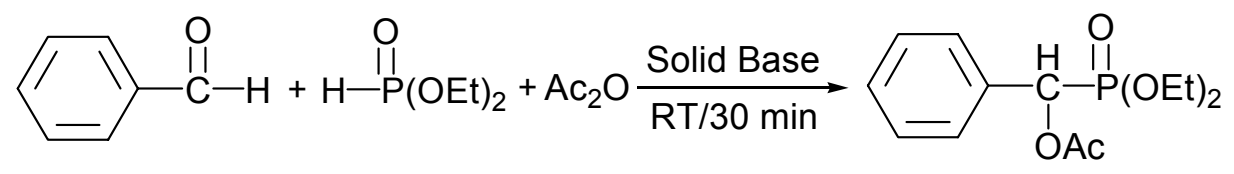

\section{Scheme 1}

Table 1. Synthesis of 1-acetoxyphosphonates from the reaction of aldehydes with diethyl phosphite in the presence of acetic anhydride under solvent-free conditions using solid bases

\begin{tabular}{cccc}
\hline Entry & Solid Catalyst & Method $^{\mathrm{b}}$ & ${\text { Yield of } \mathbf{2 a} \%^{\mathrm{a}}}^{{ }^{2}}$ \\
\hline 1 & $\mathrm{CaO}$ & $\mathrm{A}$ & 56 \\
2 & $\mathrm{BaO}$ & $\mathrm{A}$ & 60 \\
3 & $\mathrm{MgO}$ & $\mathrm{A}$ & 70 \\
4 & $\mathrm{SiO}_{2}$ & $\mathrm{~A}$ & $<5$ \\
5 & $\mathrm{Al}_{2} \mathrm{O}_{3}(\mathrm{a})$ & $\mathrm{A}$ & 20 \\
6 & $\mathrm{Al}_{2} \mathrm{O}_{3}(\mathrm{~b})$ & $\mathrm{A}$ & 45 \\
7 & $\mathrm{~K}_{2} \mathrm{CO}_{3}$ & $\mathrm{~A}$ & 87 \\
8 & $\mathrm{Na}_{2} \mathrm{CO}_{3}$ & $\mathrm{~A}$ & 86 \\
9 & $\mathrm{SiO}_{2}$ & $\mathrm{~B}$ & 32 \\
10 & $\mathrm{Al}_{2} \mathrm{O}_{3}(\mathrm{a})$ & $\mathrm{B}$ & 56 \\
11 & $\mathrm{Al}_{2} \mathrm{O}_{3}(\mathrm{~b})$ & $\mathrm{B}$ & 75 \\
\hline
\end{tabular}

${ }^{\mathrm{a}}$ Isolated yields

${ }^{\mathrm{b}} \mathrm{A}=$ dry conditions, $1 \mathrm{~h}, \mathrm{RT}$; $\mathrm{B}=$ microwave, 4 min.

In all the reactions we report in this paper, cleavage of the $\mathrm{C}-\mathrm{P}$ bond of the phosphonates was not detected and the conversion of the substrates to their corresponding acetoxy compounds was clean. Work-up of the reaction mixture is very easy and gives highly pure products, which do not need further purification.

In summary, in this paper we describe a simple procedure for the high-yielding synthesis of a variety of diethyl $\alpha$-acetoxyphosphonates by one-pot reaction of aldehydes with diethyl phosphite in the presence of acetic anhydride using magnesia. Simple work-up, solvent-free condition, fast reaction rates, mild reaction conditions, good to high yields, and the clean reactions with no tar formation make this method an attractive and a useful contribution to present methodologies.

\section{Experimental Section}

General Procedures. All chemicals were commercial products and distilled or recrystallized before use. NMR spectra were taken with a 250 Bruker Avance instrument with the chemical 
shifts being reported as $\delta$ ppm and couplings expressed in Hertz. The chemical shift data for each signal in ${ }^{1} \mathrm{H}$ NMR are given in units of $\delta$ relative to $\mathrm{CHCl}_{3}(\delta=7.26)$ for $\mathrm{CDCl}_{3}$ solution. For ${ }^{13} \mathrm{C}$ NMR spectra, the chemical shifts in $\mathrm{CDCl}_{3}$ and DMSO are recorded relative to the $\mathrm{CDCl}_{3}$ resonance $(\delta=77.0)$. The chemical shifts of ${ }^{31} \mathrm{P}$ are recorded relative to external $85 \% \mathrm{H}_{3} \mathrm{PO}_{4}$ $(\delta=0)$ with broad-band ${ }^{1} \mathrm{H}$ decoupling. A kitchen-type Samsung microwave oven, model number CE290DN, was used in experiments for method B. Silica gel column chromatography was carried out with Silica gel 100 (Merck No. 10184). Merck Silica-gel 60 F254 plates (No. 5744) were used for the preparative TLC.

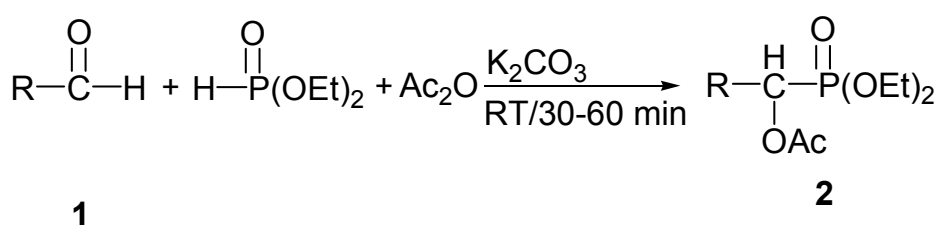

\section{Scheme 2}

Table 2. Synthesis of 1-acetoxyphosphonates from the reaction of aldehydes with diethyl phosphite in the presence of acetic anhydride under solvent-free conditions using solid potassium carbonate as base

\begin{tabular}{lllll}
\hline $\mathbf{2}$ & $\mathrm{R}$ & Reaction time (min) & Yield $\%^{\mathrm{a}}$ & $\begin{array}{l}{ }^{31} \mathrm{P} \mathrm{NMR} \\
\delta(\mathrm{ppm})\end{array}$ \\
\hline $\mathrm{A}$ & $\mathrm{Ph}-$ & 30 & 87 & 17.82 \\
$\mathrm{~B}$ & $p-\mathrm{MeOC}_{6} \mathrm{H}_{4^{-}}$ & 60 & 80 & 18.22 \\
$\mathrm{C}$ & $p-\mathrm{MeC}_{6} \mathrm{H}_{4^{-}}$ & 45 & 70 & 18.09 \\
$\mathrm{D}$ & $p-\mathrm{ClC}_{6} \mathrm{H}_{4^{-}}$ & 30 & 85 & 17.20 \\
$\mathrm{E}$ & $p-\mathrm{BrC}_{6} \mathrm{H}_{4^{-}}$ & 30 & 75 & 17.15 \\
$\mathrm{~F}$ & $m-\mathrm{ClC}_{6} \mathrm{H}_{4^{-}}$ & 30 & 80 & 16.92 \\
$\mathrm{G}$ & $m-\mathrm{MeOC}_{6} \mathrm{H}_{4^{-}}$ & 30 & 90 & 17.76 \\
$\mathrm{H}$ & $m-\mathrm{BrC}_{6} \mathrm{H}_{4}-$ & 45 & 75 & 17.03 \\
$\mathrm{I}$ & $m-\mathrm{MeC}_{6} \mathrm{H}_{4^{-}}$ & 45 & 71 & 18.00 \\
$\mathrm{~J}$ & $o-\mathrm{ClC} \mathrm{H}_{4-}$ & 60 & 78 & 17.24 \\
$\mathrm{~K}$ & $o-\mathrm{MeC} 6 \mathrm{H}_{4^{-}}$ & 60 & 74 & 18.58 \\
$\mathrm{~L}$ & $\alpha-\mathrm{Naphthyl}$ & 60 & 75 & 18.13 \\
$\mathrm{M}$ & $\beta-\mathrm{Naphthyl}$ & 45 & 70 & 17.82 \\
$\mathrm{~N}$ & $\mathrm{Ph}-\mathrm{CH}=\mathrm{CH}-$ & 30 & 70 & 6.72 \\
\hline
\end{tabular}

${ }^{\mathrm{a}}$ Isolated yields 
Synthesis of 1-acetoxyphosphonates from the reaction of aldehydes with diethyl phosphite in the presence of acetic anhydride under solvent-free conditions using solid potassium carbonate as base

This solvent-free reaction is operationally simple. Potassium carbonate $(3 \mathrm{~g})$ was added to a stirred mixture of diethyl phosphite $(0.01 \mathrm{~mol})$ and aldehyde $(0.01 \mathrm{~mol})$ at room temperature and stirred for $10 \mathrm{~min}$. Acetic anhydride $(0.03 \mathrm{~mol})$ was added to this mixture which was then stirred for 30-60 min at room temperature. The mixture was washed with ethyl acetate $(4 \times 50 \mathrm{~mL})$, dried with $\mathrm{CaCl}_{2}$, and the solvent evaporated to give crude product. Chromatography on silica gel with EtOAc/n-hexane (1:9 to 4:6) and evaporation of the solvent under reduced pressure gave the pure products in $70-90 \%$ yields. All the products gave satisfactory spectral data in accordance with the assigned structures and literature reports.

Diethyl 1-acetoxyphenylmethylphosphonate (2a). ${ }^{12}$ Colorless oil; ${ }^{1} \mathrm{H}$ NMR $\left(\mathrm{CDCl}_{3} / \mathrm{TMS}\right.$ $250 \mathrm{MHz}): 1.13-1.32(6 \mathrm{H}, \mathrm{m}), 2.09$ (3H, d, $J=2 \mathrm{~Hz}), 3.75-4.10(4 \mathrm{H}, \mathrm{m}), 6.06$ (1H, d, $J=13.5 \mathrm{~Hz})$, 7.21-7.43 (5H, m). ${ }^{31} \mathrm{P} \mathrm{NMR}\left(\mathrm{CDCl}_{3} / \mathrm{H}_{3} \mathrm{PO}_{4}, 101.2 \mathrm{MHz}\right): 17.82 \mathrm{ppm} ;{ }^{13} \mathrm{C} \mathrm{NMR}\left(\mathrm{CDCl}_{3} / \mathrm{TMS}\right.$, $62.9 \mathrm{MHz}): 16.2\left(\mathrm{~d}, J_{\mathrm{PC}}=6.3 \mathrm{~Hz}\right), 16.3\left(\mathrm{~d}, J_{\mathrm{PC}}=6.3 \mathrm{~Hz}\right), 20.8,63.1-63.3,67.6\left(\mathrm{~d}, J_{\mathrm{PC}}=169.8\right.$ $\mathrm{Hz}), 127.8\left(\mathrm{~d}, J_{\mathrm{PC}}=5.7 \mathrm{~Hz}\right), 128.4\left(\mathrm{~d}, J_{\mathrm{PC}}=2.5 \mathrm{~Hz}\right), 128.6\left(\mathrm{~d}, J_{\mathrm{PC}}=2.5 \mathrm{~Hz}\right), 133.4\left(\mathrm{~d}, J_{\mathrm{PC}}=1.9 \mathrm{~Hz}\right)$, $169.3\left(\mathrm{~d}, J_{\mathrm{PC}}=9.0 \mathrm{~Hz}\right)$.

Diethyl 1-acetoxy(4-methoxyphenyl)methylphosphonate (2b). ${ }^{12}$ Colorless oil; ${ }^{1} \mathrm{H}$ NMR $\left(\mathrm{CDCl}_{3} / \mathrm{TMS}-250 \mathrm{MHz}\right): 1.13-1.22(6 \mathrm{H}, \mathrm{m}), 2.09(3 \mathrm{H}, \mathrm{d}, J=1.2 \mathrm{~Hz}), 3.77(3 \mathrm{H}, \mathrm{d}, J=1.5 \mathrm{~Hz})$, 3.85-4.15 (4H, m), $6.05(1 \mathrm{H}, \mathrm{d}, J=13.0 \mathrm{~Hz}), 6.86(2 \mathrm{H}, \mathrm{d}, J=7.7 \mathrm{~Hz}), 7.41(2 \mathrm{H}, \mathrm{d}, J=7.7 \mathrm{~Hz}) .{ }^{31} \mathrm{P}$ NMR $\left(\mathrm{CDCl}_{3} / \mathrm{H}_{3} \mathrm{PO}_{4}, 101.2 \mathrm{MHz}\right): 18.22 \mathrm{ppm} ;{ }^{13} \mathrm{C} \mathrm{NMR}\left(\mathrm{CDCl}_{3} / \mathrm{TMS}, 62.9 \mathrm{MHz}\right): 16.2$ (d, $\left.J_{\mathrm{PC}}=5.8 \mathrm{~Hz}\right), 16.4\left(\mathrm{~d}, J_{\mathrm{PC}}=5.7 \mathrm{~Hz}\right), 20.9,55.2,63.1-63.3,70.0\left(\mathrm{~d}, J_{\mathrm{PC}}=172.7 \mathrm{~Hz}\right), 113.9$ (d, $\left.J_{\mathrm{PC}}=1.8 \mathrm{~Hz}\right), 125.4\left(\mathrm{~d}, J_{\mathrm{PC}}=1.8 \mathrm{~Hz}\right), 129.5\left(\mathrm{~d}, J_{\mathrm{PC}}=6.1 \mathrm{~Hz}\right), 159.9\left(\mathrm{~d}, J_{\mathrm{PC}}=2.5 \mathrm{~Hz}\right), 169.3(\mathrm{~d}$, $J_{\mathrm{PC}}=9.1 \mathrm{~Hz}$ ).

Diethyl 1-acetoxy-(4-methylphenyl)methylphosphonate (2c). ${ }^{12}$ Colorless oil; ${ }^{1} \mathrm{H}$ NMR $\left(\mathrm{CDCl}_{3} / \mathrm{TMS}-250 \mathrm{MHz}\right): 1.15-1.30(6 \mathrm{H}, \mathrm{m}), 2.14(3 \mathrm{H}, \mathrm{s}), 2.32(3 \mathrm{H}, \mathrm{s}), 3.85-4.15(4 \mathrm{H}, \mathrm{m})$, $6.09(1 \mathrm{H}, \mathrm{d}, J=13.2 \mathrm{~Hz}), 7.16(2 \mathrm{H}, \mathrm{d}, J=8.0 \mathrm{~Hz}), 7.38(2 \mathrm{H}, \mathrm{d}, J=8.0 \mathrm{~Hz}) .{ }^{31} \mathrm{P} \mathrm{NMR}\left(\mathrm{CDCl}_{3} /\right.$ $\left.\mathrm{H}_{3} \mathrm{PO}_{4}, 101.2 \mathrm{MHz}\right): 18.09 \mathrm{ppm} ;{ }^{13} \mathrm{C} \mathrm{NMR}\left(\mathrm{CDCl}_{3} / \mathrm{TMS}, 62.9 \mathrm{MHz}\right): 16.2\left(\mathrm{~d}, J_{\mathrm{PC}}=5.8 \mathrm{~Hz}\right), 16.4$ $\left(\mathrm{d}, J_{\mathrm{PC}}=5.7 \mathrm{~Hz}\right), 20.9,21.2,63.2\left(\mathrm{~d}, J_{\mathrm{PC}}=6.8 \mathrm{~Hz}\right), 70.3\left(\mathrm{~d}, J_{\mathrm{PC}}=171.0 \mathrm{~Hz}\right), 127.9\left(\mathrm{~d}, J_{\mathrm{PC}}=6.0 \mathrm{~Hz}\right)$, $129.1\left(\mathrm{~d}, J_{\mathrm{PC}}=2.1 \mathrm{~Hz}\right), 130.4\left(\mathrm{~d}, J_{\mathrm{PC}}=2.0 \mathrm{~Hz}\right), 138.6\left(\mathrm{~d}, J_{\mathrm{PC}}=3.0 \mathrm{~Hz}\right), 169.3\left(\mathrm{~d}, J_{\mathrm{PC}}=9.0 \mathrm{~Hz}\right)$.

Diethyl 1-acetoxy (4-chlorophenyl)methylphosphonate (2d). ${ }^{12}$ Colorless oil; ${ }^{1} \mathrm{H}$ NMR $\left(\mathrm{CDCl}_{3} / \mathrm{TMS}, 250 \mathrm{MHz}\right)$ : 1.13-1.30 $(6 \mathrm{H}, \mathrm{m}), 2.13(3 \mathrm{H}, \mathrm{s}), 3.85-4.18(4 \mathrm{H}, \mathrm{m}), 6.05(1 \mathrm{H}, \mathrm{d}$, $J=13.7 \mathrm{~Hz}), 7.30(2 \mathrm{H}, \mathrm{d}, J=8.5 \mathrm{~Hz}), 7.39(2 \mathrm{H}, \mathrm{d}, J=8.5 \mathrm{~Hz}) .{ }^{31} \mathrm{P} \mathrm{NMR}\left(\mathrm{CDCl}_{3} / \mathrm{H}_{3} \mathrm{PO}_{4}, 101.2\right.$ MHz):17.20 ppm; ${ }^{13} \mathrm{C} \mathrm{NMR}\left(\mathrm{CDCl}_{3} / \mathrm{TMS}, 62.9 \mathrm{MHz}\right): 16.2\left(\mathrm{~d}, J_{\mathrm{PC}}=6.3 \mathrm{~Hz}\right), 16.3\left(\mathrm{~d}, J_{\mathrm{PC}}=6.3 \mathrm{~Hz}\right)$, 20.6, 63.2-63.3, $69.8\left(\mathrm{~d}, J_{\mathrm{PC}}=171.1 \mathrm{~Hz}\right), 128.6\left(\mathrm{~d}, J_{\mathrm{PC}}=1.9 \mathrm{~Hz}\right), 129.2\left(\mathrm{~d}, J_{\mathrm{PC}}=5.7 \mathrm{~Hz}\right), 132.2(\mathrm{~d}$, $\left.J_{\mathrm{PC}}=1.9 \mathrm{~Hz}\right), 134.6\left(\mathrm{~d}, J_{\mathrm{PC}}=3.1 \mathrm{~Hz}\right), 168.9\left(\mathrm{~d}, J_{\mathrm{PC}}=8.8 \mathrm{~Hz}\right)$.

Diethyl 1-acetoxy-(4-bromophenyl)methylphosphonate (2e). Colorless oil; ${ }^{1} \mathrm{H}$ NMR $\left(\mathrm{CDCl}_{3} / \mathrm{TMS}, 250 \mathrm{MHz}\right)$ : 1.15-1.30 $(6 \mathrm{H}, \mathrm{m}), 2.15(3 \mathrm{H}, \mathrm{s}), 3.80-4.08(4 \mathrm{H}, \mathrm{m}), 6.03(1 \mathrm{H}, \mathrm{d}$, $J=13.7 \mathrm{~Hz}), 7.33(2 \mathrm{H}, \mathrm{d}, J=8.2 \mathrm{~Hz}), 7.47(2 \mathrm{H}, \mathrm{d}, J=8.2 \mathrm{~Hz}) .{ }^{31} \mathrm{P} \mathrm{NMR}\left(\mathrm{CDCl}_{3} / \mathrm{H}_{3} \mathrm{PO}_{4}, 101.2\right.$ $\mathrm{MHz}): 17.15 \mathrm{ppm} ;{ }^{13} \mathrm{C} \mathrm{NMR}\left(\mathrm{CDCl}_{3} / \mathrm{TMS}, 62.9 \mathrm{MHz}\right): 16.3\left(\mathrm{~d}, J_{\mathrm{PC}}=6.3 \mathrm{~Hz}\right), 16.4\left(\mathrm{~d}, J_{\mathrm{PC}}=6.3 \mathrm{~Hz}\right)$, 
20.8, 63.3-63.4, $69.8\left(\mathrm{~d}, J_{\mathrm{PC}}=170.6 \mathrm{~Hz}\right), 122.9\left(\mathrm{~d}, J_{\mathrm{PC}}=3.6 \mathrm{~Hz}\right), 129.5\left(\mathrm{~d}, J_{\mathrm{PC}}=5.8 \mathrm{~Hz}\right), 131.6(\mathrm{~d}$, $\left.J_{\mathrm{PC}}=2.1 \mathrm{~Hz}\right), 132.6\left(\mathrm{~d}, J_{\mathrm{PC}}=2.0 \mathrm{~Hz}\right), 169.2\left(\mathrm{~d}, J_{\mathrm{PC}}=9.0 \mathrm{~Hz}\right)$. Anal. Calcd for $\mathrm{C}_{13} \mathrm{H}_{18} \mathrm{BrO}_{5} \mathrm{P}: \mathrm{C}$, 42.74; H, 4.93. Found: C, 42.8; H, 5.1.

Diethyl 1-acetoxy-(3-chlorophenyl)methylphosphonate (2f). ${ }^{12}$ Colorless oil; ${ }^{1} \mathrm{H} \quad \mathrm{NMR}$ $\left(\mathrm{CDCl}_{3} / \mathrm{TMS}, 250 \mathrm{MHz}\right)$ : 1.10-1.25 $(6 \mathrm{H}, \mathrm{m}), 2.10(3 \mathrm{H}, \mathrm{s}), 3.85-4.18(4 \mathrm{H}, \mathrm{m}), 6.05(1 \mathrm{H}, \mathrm{d}$, $J=14.0 \mathrm{~Hz}), 7.15-7.45$ (4H, m). ${ }^{31} \mathrm{P}$ NMR $\left(\mathrm{CDCl}_{3} / \mathrm{H}_{3} \mathrm{PO}_{4}, 101.2 \mathrm{MHz}\right): 16.92 \mathrm{ppm} ;{ }^{13} \mathrm{C} \mathrm{NMR}$ $\left(\mathrm{CDCl}_{3} / \mathrm{TMS}, 62.9 \mathrm{MHz}\right): 16.1\left(\mathrm{~d}, J_{\mathrm{PC}}=5.6 \mathrm{~Hz}\right), 16.2\left(\mathrm{~d}, J_{\mathrm{PC}}=5.6 \mathrm{~Hz}\right), 20.6,63.2\left(\mathrm{~d}, J_{\mathrm{PC}}=6.3 \mathrm{~Hz}\right)$, $63.3\left(\mathrm{~d}, J_{\mathrm{PC}}=6.3 \mathrm{~Hz}\right), 69.8\left(\mathrm{~d}, J_{\mathrm{PC}}=169.8 \mathrm{~Hz}\right), 125.9\left(\mathrm{~d}, J_{\mathrm{PC}}=5.6 \mathrm{~Hz}\right), 127.7\left(\mathrm{~d}, J_{\mathrm{PC}}=5.7 \mathrm{~Hz}\right), 128.7$ $\left(\mathrm{d}, J_{\mathrm{PC}}=2.5 \mathrm{~Hz}\right), 129.6,134.3,135.6,168.9\left(\mathrm{~d}, J_{\mathrm{PC}}=8.2 \mathrm{~Hz}\right)$.

Diethyl 1-acetoxy-(3-methoxyphenyl)-methylphosphonate (2g). ${ }^{37}$ Colorless oil; ${ }^{1} \mathrm{H}$ NMR $\left(\mathrm{CDCl}_{3} / \mathrm{TMS}, 250 \mathrm{MHz}\right)$ : 1.15-1.29 (6H, m), $2.14(3 \mathrm{H}, \mathrm{s}), 3.78(3 \mathrm{H}, \mathrm{d}, J=1.5 \mathrm{~Hz}), 3.85-4.15$ (4H, m), 6.07 (1H, d, J=13.7 Hz), 6.78-7.28 (4H, m). ${ }^{31} \mathrm{P} \mathrm{NMR}\left(\mathrm{CDCl}_{3} / \mathrm{H}_{3} \mathrm{PO}_{4}, 101.2 \mathrm{MHz}\right)$ : $17.76 \mathrm{ppm} ;{ }^{13} \mathrm{C} \mathrm{NMR}\left(\mathrm{CDCl}_{3} / \mathrm{TMS}, 62.9 \mathrm{MHz}\right): 16.2$ (d, $\left.J_{\mathrm{PC}}=5.8 \mathrm{~Hz}\right), 16.4\left(\mathrm{~d}, J_{\mathrm{PC}}=5.9 \mathrm{~Hz}\right), 20.9$, $55.2,63.3\left(\mathrm{~d}, J_{\mathrm{PC}}=5.9 \mathrm{~Hz}\right), 70.3\left(\mathrm{~d}, J_{\mathrm{PC}}=170.1 \mathrm{~Hz}\right), 113.1\left(\mathrm{~d}, J_{\mathrm{PC}}=5.5 \mathrm{~Hz}\right), 114.3\left(\mathrm{~d}, J_{\mathrm{PC}}=2.7 \mathrm{~Hz}\right)$, $120.1\left(\mathrm{~d}, J_{\mathrm{PC}}=5.8 \mathrm{~Hz}\right), 129.5,134.8,159.5,169.2\left(\mathrm{~d}, J_{\mathrm{PC}}=9.1 \mathrm{~Hz}\right)$.

Diethyl 1-acetoxy-(3-bromophenyl)methylphosphonate (2h). Colorless oil; ${ }^{1} \mathrm{H} \quad \mathrm{NMR}$ $\left(\mathrm{CDCl}_{3} / \mathrm{TMS}-250 \mathrm{MHz}\right): 1.10-1.25(6 \mathrm{H}, \mathrm{m}), 2.10(2 \mathrm{H}, \mathrm{d}, J=2.2 \mathrm{~Hz}), 3.85-4.18(4 \mathrm{H}, \mathrm{m}), 6.03$ $(1 \mathrm{H}, \mathrm{d}, J=13.7 \mathrm{~Hz}), 7.10-7.58(4 \mathrm{H}, \mathrm{m}) .{ }^{31} \mathrm{P} \mathrm{NMR}\left(\mathrm{CDCl}_{3} / \mathrm{H}_{3} \mathrm{PO}_{4}, 101.2 \mathrm{MHz}\right): 17.03 \mathrm{ppm} ;{ }^{13} \mathrm{C}$ NMR (CDCl 3 /TMS, 62.9 MHz):16.1 (d, $\left.J_{\mathrm{PC}}=5.7 \mathrm{~Hz}\right), 16.2\left(\mathrm{~d}, J_{\mathrm{PC}}=5.7 \mathrm{~Hz}\right), 20.6,63.2\left(\mathrm{~d}, J_{\mathrm{PC}}=7.0\right.$ $\mathrm{Hz}), 63.3\left(\mathrm{~d}, J_{\mathrm{PC}}=7.0 \mathrm{~Hz}\right), 69.4\left(\mathrm{~d}, J_{\mathrm{PC}}=169.8 \mathrm{~Hz}\right), 122.2\left(\mathrm{~d}, J_{\mathrm{PC}}=2.5 \mathrm{~Hz}\right), 126.3\left(\mathrm{~d}, J_{\mathrm{PC}}=5.0 \mathrm{~Hz}\right)$, $129.8\left(\mathrm{~d}, J_{\mathrm{PC}}=1.9 \mathrm{~Hz}\right), 130.4\left(\mathrm{~d}, J_{\mathrm{PC}}=5.7 \mathrm{~Hz}\right), 131.5\left(\mathrm{~d}, J_{\mathrm{PC}}=2.5 \mathrm{~Hz}\right), 135.6\left(\mathrm{~d}, J_{\mathrm{PC}}=1.9 \mathrm{~Hz}\right), 168.8$ $\left(\mathrm{d}, J_{\mathrm{PC}}=8.8 \mathrm{~Hz}\right.$ ). Anal. Calcd for $\mathrm{C}_{13} \mathrm{H}_{18} \mathrm{BrO}_{5} \mathrm{P}: \mathrm{C}, 42.74 ; \mathrm{H}, 4.93$. Found: C, 42.6; H, 5.1.

Diethyl 1-acetoxy-(3-methylphenyl)methylphosphonate (2i). Colorless oil; ${ }^{1} \mathrm{H}$ NMR $\left(\mathrm{CDCl}_{3} / \mathrm{TMS}-250 \mathrm{MHz}\right): 1.15-1.30(6 \mathrm{H}, \mathrm{m}), 2.16(3 \mathrm{H}, \mathrm{s}), 2.34(3 \mathrm{H}, \mathrm{s}), 3.85-4.15(4 \mathrm{H}, \mathrm{m})$, 6.09 (1H, d, $J=13.2 \mathrm{~Hz}), 7.10-7.45$ (4H, m). ${ }^{31} \mathrm{P}$ NMR $\left(\mathrm{CDCl}_{3} / \mathrm{H}_{3} \mathrm{PO}_{4}, 101.2 \mathrm{MHz}\right): 18.00 \mathrm{ppm}$; ${ }^{13} \mathrm{C} \mathrm{NMR}\left(\mathrm{CDCl}_{3} / \mathrm{TMS}, 62.9 \mathrm{MHz}\right): 16.2\left(\mathrm{~d}, J_{\mathrm{PC}}=5.9 \mathrm{~Hz}\right), 16.4\left(\mathrm{~d}, J_{\mathrm{PC}}=5.9 \mathrm{~Hz}\right), 20.9,21.4,63.1-$ 63.3, $70.5\left(\mathrm{~d}, J_{\mathrm{PC}}=170.1 \mathrm{~Hz}\right), 124.9\left(\mathrm{~d}, J_{\mathrm{PC}}=5.8 \mathrm{~Hz}\right), 128.3\left(\mathrm{~d}, J_{\mathrm{PC}}=2.2 \mathrm{~Hz}\right), 128.5\left(\mathrm{~d}, J_{\mathrm{PC}}=5.9 \mathrm{~Hz}\right)$, $129.5\left(\mathrm{~d}, J_{\mathrm{PC}}=2.8 \mathrm{~Hz}\right), 133.3,138.1,169.3\left(\mathrm{~d}, J_{\mathrm{PC}}=8.9 \mathrm{~Hz}\right)$. Anal. Calcd for $\mathrm{C}_{14} \mathrm{H}_{21} \mathrm{O}_{5} \mathrm{P}: \mathrm{C}, 56.00$; H, 7.00. Found: C, 56.2; H, 7.2.

Diethyl 1-acetoxy-(2-chlorophenyl)methylphosphonate $\quad(\mathbf{2 j}) .{ }^{12}$ Colorless oil; ${ }^{1} \mathrm{H} \quad \mathrm{NMR}$ $\left(\mathrm{CDCl}_{3} / \mathrm{TMS}-250 \mathrm{MHz}\right): 1.15(3 \mathrm{H}, \mathrm{t}, J=7.0 \mathrm{~Hz}), 1.28(3 \mathrm{H}, \mathrm{t}, J=7.0 \mathrm{~Hz}), 2.12(3 \mathrm{H}, \mathrm{s}), 3.80-4.21$ $(4 \mathrm{H}, \mathrm{m}), 6.58(1 \mathrm{H}, \mathrm{d}, J=13.8 \mathrm{~Hz}), 7.15-7.65(4 \mathrm{H}, \mathrm{m}) .{ }^{31} \mathrm{P} \mathrm{NMR}\left(\mathrm{CDCl}_{3} / \mathrm{H}_{3} \mathrm{PO}_{4}, 101.2\right.$ $\mathrm{MHz}): 17.24 \mathrm{ppm} ;{ }^{13} \mathrm{C} \mathrm{NMR}\left(\mathrm{CDCl}_{3} / \mathrm{TMS}, 62.9 \mathrm{MHz}\right): 16.2\left(\mathrm{~d}, J_{\mathrm{PC}}=5.7 \mathrm{~Hz}\right), 16.3\left(\mathrm{~d}, J_{\mathrm{PC}}=5.7 \mathrm{~Hz}\right)$, 20.7, $63.2\left(\mathrm{~d}, J_{\mathrm{PC}}=6.6 \mathrm{~Hz}\right), 66.8\left(\mathrm{~d}, J_{\mathrm{PC}}=172.3 \mathrm{~Hz}\right), 127.0,129.4,129.6\left(\mathrm{~d}, J_{\mathrm{PC}}=3.8 \mathrm{~Hz}\right), 129.8(\mathrm{~d}$, $\left.J_{\mathrm{PC}}=2.8 \mathrm{~Hz}\right), 131.9,133.6\left(\mathrm{~d}, J_{\mathrm{PC}}=7.9 \mathrm{~Hz}\right), 168.9\left(\mathrm{~d}, J_{\mathrm{PC}}=9.6 \mathrm{~Hz}\right)$.

Diethyl 1-acetoxy-(2-methylphenyl)methylphosphonate (2k). ${ }^{6}$ Colorless oil; ${ }^{1} \mathrm{H}$ NMR $\left(\mathrm{CDCl}_{3} / \mathrm{TMS}-250 \mathrm{MHz}\right): 1.10-1.35(6 \mathrm{H}, \mathrm{m}), 2.14(3 \mathrm{H}, \mathrm{s}), 2.48(3 \mathrm{H}, \mathrm{s}), 3.85-4.18(4 \mathrm{H}, \mathrm{m})$, $6.36(1 \mathrm{H}, \mathrm{d}, J=13.7 \mathrm{~Hz}), 7.10-7.65(4 \mathrm{H}, \mathrm{m}) .{ }^{31} \mathrm{P} \mathrm{NMR}\left(\mathrm{CDCl}_{3} / \mathrm{H}_{3} \mathrm{PO}_{4}, 101.2 \mathrm{MHz}\right): 18.58 \mathrm{ppm}$; ${ }^{13} \mathrm{C} \mathrm{NMR}\left(\mathrm{CDCl}_{3} / \mathrm{TMS}, 62.9 \mathrm{MHz}\right): 16.2\left(\mathrm{~d}, J_{\mathrm{PC}}=5.6 \mathrm{~Hz}\right), 16.4\left(\mathrm{~d}, J_{\mathrm{PC}}=5.6 \mathrm{~Hz}\right), 19.6,20.92,63.2$ 
$\left(\mathrm{d}, J_{\mathrm{PC}}=6.9 \mathrm{~Hz}\right), 67.0\left(\mathrm{~d}, J_{\mathrm{PC}}=171.7 \mathrm{~Hz}\right), 126.2,128.1\left(\mathrm{~d}, J_{\mathrm{PC}}=4.4 \mathrm{~Hz}\right), 128.6,130.3,132.1,136.7$ $\left(\mathrm{d}, J_{\mathrm{PC}}=7.5 \mathrm{~Hz}\right), 169.3\left(\mathrm{~d}, J_{\mathrm{PC}}=10.0 \mathrm{~Hz}\right)$.

Diethyl 1-acetoxy-(1-naphthyl)methylphosphonate $\quad$ (2I). ${ }^{12} \quad$ Colorless $\quad$ oil; $\quad{ }^{1} \mathrm{H} \quad \mathrm{NMR}$ $\left(\mathrm{CDCl}_{3} / \mathrm{TMS}, 250 \mathrm{MHz}\right): 0.95(3 \mathrm{H}, \mathrm{t}, J=7.0 \mathrm{~Hz}), 1.15(3 \mathrm{H}, \mathrm{t}, J=7.0 \mathrm{~Hz}), 2.10(3 \mathrm{H}, \mathrm{s}), 3.58-4.21$ $(4 \mathrm{H}, \mathrm{m}), 6.94(1 \mathrm{H}, \mathrm{d}, J=14.2 \mathrm{~Hz}), 7.35-8.0(6 \mathrm{H}, \mathrm{m}), 8.22(1 \mathrm{H}, \mathrm{d}, J=8.5 \mathrm{~Hz}) .{ }^{31} \mathrm{P}$ NMR $\left(\mathrm{CDCl}_{3} /\right.$ $\left.\mathrm{H}_{3} \mathrm{PO}_{4}, 101.2 \mathrm{MHz}\right): 18.13 \mathrm{ppm} ;{ }^{13} \mathrm{C} \mathrm{NMR}\left(\mathrm{CDCl}_{3} / \mathrm{TMS}, 62.9 \mathrm{MHz}\right): 16.1\left(\mathrm{~d}, J_{\mathrm{PC}}=5.6 \mathrm{~Hz}\right), 16.3$ $\left(\mathrm{d}, J_{\mathrm{PC}}=5.6 \mathrm{~Hz}\right), 20.8,63.2\left(\mathrm{~d}, J_{\mathrm{PC}}=6.6 \mathrm{~Hz}\right), 67.1\left(\mathrm{~d}, J_{\mathrm{PC}}=172.0 \mathrm{~Hz}\right), 123.7,125.2\left(\mathrm{~d}, J_{\mathrm{PC}}=3.8 \mathrm{~Hz}\right)$, $125.8,126.5,126.7\left(\mathrm{~d}, J_{\mathrm{PC}}=5.9 \mathrm{~Hz}\right), 128.6,129.4,129.7,133.6\left(\mathrm{~d}, J_{\mathrm{PC}}=5.5 \mathrm{~Hz}\right), 133.6,169.2(\mathrm{~d}$, $\left.J_{\mathrm{PC}}=9.0 \mathrm{~Hz}\right)$.

Diethyl 1-acetoxy-(2-naphthyl)methylphosphonate $\quad(\mathbf{2 m}){ }^{12}$ Colorless $\quad$ oil; ${ }^{1} \mathrm{H} \quad \mathrm{NMR}$ $\left(\mathrm{CDCl}_{3} / \mathrm{TMS}, 250 \mathrm{MHz}\right): 1.10(3 \mathrm{H}, \mathrm{t}, J=7.0 \mathrm{~Hz}), 1.17(3 \mathrm{H}, \mathrm{t}, J=7.0 \mathrm{~Hz}), 2.10(3 \mathrm{H}, \mathrm{s}), 3.75-4.15$ $(4 \mathrm{H}, \mathrm{m}), 6.28(1 \mathrm{H}, \mathrm{d}, J=13.7 \mathrm{~Hz}), 7.30-7.95(7 \mathrm{H}, \mathrm{m}) .{ }^{31} \mathrm{P} \mathrm{NMR}\left(\mathrm{CDCl}_{3} / \mathrm{H}_{3} \mathrm{PO}_{4}, 101.2\right.$ MHz):17.82 ppm; $\left.{ }^{13} \mathrm{C} \mathrm{NMR} \mathrm{(CDCl} / \mathrm{TMS}, 62.9 \mathrm{MHz}\right): 16.2\left(\mathrm{~d}, J_{\mathrm{PC}}=5.7 \mathrm{~Hz}\right), 16.3\left(\mathrm{~d}, J_{\mathrm{PC}}=5.7\right.$ $\mathrm{Hz}), 20.8,63.2-63.3,70.5\left(\mathrm{~d}, J_{\mathrm{PC}}=169.8 \mathrm{~Hz}\right), 125.2\left(\mathrm{~d}, J_{\mathrm{PC}}=4.4 \mathrm{~Hz}\right), 126.3,126.5,127.3(\mathrm{~d}$, $\left.J_{\mathrm{PC}}=7.5 \mathrm{~Hz}\right), 127.6,128.1,128.2\left(\mathrm{~d}, J_{\mathrm{PC}}=1.9 \mathrm{~Hz}\right), 130.9\left(\mathrm{~d}, J_{\mathrm{PC}}=2.5 \mathrm{~Hz}\right), 132.9\left(\mathrm{~d}, J_{\mathrm{PC}}=2.5 \mathrm{~Hz}\right)$, $133.2\left(\mathrm{~d}, J_{\mathrm{PC}}=2.5 \mathrm{~Hz}\right), 169.2\left(\mathrm{~d}, J_{\mathrm{PC}}=8.8 \mathrm{~Hz}\right)$.

Diethyl 1-acetoxy-(2-phenylethenyl)methylphosphonate (2n). ${ }^{12}$ Colorless oil; ${ }^{1} \mathrm{H}$ NMR $\left(\mathrm{CDCl}_{3} / \mathrm{TMS}-250 \mathrm{MHz}\right): 1.15-1.35(6 \mathrm{H}, \mathrm{m}), 2.11(3 \mathrm{H}, \mathrm{s}), 3.95-4.30(4 \mathrm{H}, \mathrm{m}), 5.80(1 \mathrm{H}, \mathrm{ddd}, J$ $=1.0, J=7.2$ and $J=14.0 \mathrm{~Hz}), 6.10-6.25(1 \mathrm{H}, \mathrm{m}), 6.58(1 \mathrm{H}, \mathrm{dd}, J=3.5$ and $J=15.7 \mathrm{~Hz}), 7.15-7.45$ $(5 \mathrm{H}, \mathrm{m}) .{ }^{31} \mathrm{P}$ NMR $\left(\mathrm{CDCl}_{3} / \mathrm{H}_{3} \mathrm{PO}_{4}, 101.2 \mathrm{MHz}\right): 6.72 \mathrm{ppm} ;{ }^{13} \mathrm{C}$ NMR $\left(\mathrm{CDCl}_{3} / \mathrm{TMS}, 62.9 \mathrm{MHz}\right)$ : $16.2-16.3,20.8,63.1-63.4,69.2\left(\mathrm{~d}, J_{\mathrm{PC}}=171.4 \mathrm{~Hz}\right), 120.1\left(\mathrm{~d}, J_{\mathrm{PC}}=4.5 \mathrm{~Hz}\right) 126.7\left(\mathrm{~d}, J_{\mathrm{PC}}=1.3 \mathrm{~Hz}\right)$, $128.6,135.0,135.6\left(\mathrm{~d}, J_{\mathrm{PC}}=2.8 \mathrm{~Hz}\right), 169.2\left(\mathrm{~d}, J_{\mathrm{PC}}=7.9 \mathrm{~Hz}\right)$.

\section{Acknowledgements}

The Institute for Advanced Studies in Basic Sciences (IASBS) is thanked for supporting this work.

\section{References and Notes}

1. (a) Fadel, A.; Yefash, R.; Saluan, J. Synthesis 1987, 37. (b) Rosini, G.; Galarini, R.; Marotta, E.; Righi, R. J. Org. Chem. 1990, 55, 781. (c) Kodomari, M.; Sakamoto, T.; Yoshitomi, S. J. Chem. Soc. Chem. Commun. 1990, 701. (d) Kropp, P. J.; Daus, K. A.; Crawford, S. D.; Tubergren, M. W.; Kepler, K. D.; Craig, S. L.; Wilson, V. P. J. Am. Chem. Soc. 1990, 112, 7433. (e) Hondrogiannis, G.; Pagni, R. M.; Kabalka, G. W.; Anisoki, P.; Kurt, R. Tetrahedron Lett. 1990, 31, 5433. (f) Kaboudin B.; Saadati, F. Tetrahedron Lett. 2007, 48, 2829. (g) Pauter, F.; Daudon, M. Tetrahedron Lett. 1991, 32, 1457. (h) Kaboudin, B.; 
Navaee K. Heterocycles 2001, 55, 1443. (i) Kaboudin B.; Saadati, F. Heterocycles 2005, 65, 353. (j) Kaboudin, B.; Saadati, F. J. Heterocycl. Chem. 2005, 42, 699.

2. (a) Engel, R. Chem. Rev. 1977, 77, 349. (b) Prasad G. S.; Manjunath, M.; Reddy, K. R. K. K.; Reddy, O. V. S.; Reddy, C. S. Arkivoc 2006. 128.

3. (a) Dingwall, J. G.; Campbell, C. D.; Baylis, E. K. UK Patent Appl., 1542 938, 1979; Chem. Abstr. 1979, 88, 105559j. (b) Kafarski, P.; Lejczak, B.; Tyka, R.; Koba, L.; Pliszczak, E.; Wieczorek, P. J. Plant Growth Regul. 1995, 14, 199. (c) Ishiguri, Y.; Yamada, Y.; Kato, T.; Sasaki, M.; Mukai, K. Eur. Patent Appl., EP 82-301905, 1982; Chem. Abstr. 1983, 98, 102686u. (d) Kaboudin, B. Tetrahedron Lett. 2000, 41, 3169-3171. (e) Kaboudin, B. J. Chem. Res. 1999, 402. (f) Kaboudin, B. Nazari, R. J. Chem. Res. 2002, 291. (g) Kaboudin, B.; Emadi, E.; Norouzi, H. Phosphorus Sulfur and Silicon 2004, 179, 585. (h) Kaboudin, B.; Nazari, R. Tetrahedron Lett. 2001, 42, 8211. (i) Kaboudin, B.; Nazari, R. Synth. Commun. 2001, 31, 2245. (j) Kaboudin, B.; Balakrishna, M. S. Synth. Commun. 2001, 31, 2773. (k) Kaboudin, B. Tetrahedron Lett. 2002, 43, 8713. (1) Kaboudin, B. Tetrahedron Lett. 2003, 44, 1051. (m) Kaboudin, B.; Rahmani, A. Synthesis 2003, 2705. (n) Kaboudin, B.; Saadati, F. Synthesis 2004, 1249.

4. (a) Li, Y. F.; Hammerschmidt, F. Tetrahedron 1995, 51, 4933. (b) Li, Y. F.; Hammerschmidt, F. Tetrahedron: Asymmetry 1993, 4, 109. (c) Heisler, A.; Rabiller, C.; Douillard, R.; Goalou, N.; Hagele, G.; Levayer, F. Tetrahedron: Asymmetry 1993, 4, 959. (d) Khushi, T.; O’Toole, K. J.; Sime, J. T. Tetrahedron Lett. 1993, 34, 2375. (e) Zhang, Y.; Li, J. F.; Yuan, C. Y. Tetrahedron 2003, 59, 473.

5. (a) Hammerschmidt, F.; Vollenkle, H. Leibigs Ann. Chem. 1989, 577. (b) Yokomatsu, T.; Shibuya, S. Tetrahedron: Asymmetry 1992, 3, 377. (c) Baraldi, P. G.; Guarneri, M.; Moroder, F.; Pollini, G. P.; Simoni, D. Synthesis 1982, 653. (d) Maier, L. Phosphorus Sulfur Silicon Relat. Elem. 1993, 76, 119. (e) McClure, C. K.; Mishra, P. K.; Grote, C. W. J. Org. Chem. 1997, 62, 2437.

6. (a) Drescher, M.; Hammerschmidt, F.; Kahlig, H. Synthesis 1995, 1267. (b) Eidenhammer, G.; Hammerschmidt, F. Synthesis 1996, 748.

7. (a) Hilderbrand, R. L. The Role of Phosphonates in Living Systems; CRC Press: Boca Raton, F1, 1982. (b) Redmore D. Topics in Phosphorus Chemistry; Griffith, E. J., Grayson, M., Eds.; Wiley: New York, 1976; Vol. 8. (c) Pudovik, A. N.; Konovalova, I. V. Synthesis 1979, 81. (d) Wynberg, H.; Smaardijk. A. A. Tetrahedron Lett. 1983, 24, 5899. (e) Blazis, V. J.; Koeller, K. J.; Spilling, C. D. Tetrahedron: Asymmetry 1994, 5, 499. (f) Rath, N. P.; Spilling, C. D. Tetrahedron Lett. 1994, 35, 227. (g) Blazis, V. J.; Koeller, K. J.; Spilling, C. D. J. Org. Chem. 1995, 60, 931. (h) Yokomatsu, T.; Yamagishi, T.; Shibuya, S. Tetrahedron: Asymmetry 1993, 4, 1779. (i) Yokomatsu, T.; Yoshida, Y.; Shibuya, S. J. Org. Chem. 1994, 59,7930 .

8. Nesterov, L. V.; Sabirova, R. A.; Krepysheva, N. E. J. Gen. Chem. USSR (Eng Transl) 1969, 39, 1906.

9. McConnell, R. L.; Coover, H. W. Jr. J. Am. Chem. Soc. 1957, 79, 1961. 
10. Pudovik, A. N.; Gozman, I. P.; Nikitina, V. I. J. Gen. Chem. USSR (Eng Transl) 1963, 33, 3128.

11. Strepikheev, Y. A.; Kovalenko, L. V.; Batalina, A. V.; Shvetsova, L. A. Deposited Doc. 1976, VINITI: 2264; Chem. Abstr. 1978, 89, 129601.

12. (a) Firouzabadi, H.; Iranpoor, N.; Sobhani, S.; Amoozgar, Z. Synthesis 2004, 295. (b) Firouzabadi, H.; Iranpoor, N.; Sobhani, S.; Amoozgar, Z. Synthesis 2004, 1771.

13. (a) Kaboudin, B.; Rahmani, A. Org. Prep. Proced. Int. 2004, 36, 82. (b)Kaboudin, B.; Moradi, K. Tetrahedron Lett. 2005, 46, 2989. (c) Kaboudin, B.; Haghighat, H. Tetrahedron Lett. 2005, 46, 7955. (d) Kaboudin, B.; Haghighat, H.; Yokomatsu, T. J. Org. Chem. 2006, 71, 6604. (e) Kaboudin, B.; Karimi, M. Bioorg. Med. Chem. Lett. 2006, 16, 5324. (f) Kaboudin, B.; Farjadian, F. Beilstein J. Org. Chem. 2006, 2, 4. (g) Kaboudin, B.; Norouzi, H. Synthesis 2004, 2035. (h) Kaboudin, B.; Elhamifar, D.; Farjadian, F. Org. Prep. Proced. Int. 2006, 38, 412. (i) Kaboudin, B.; Norouzi, H. Tetrahedron Lett. 2004, 45, 1283. (j) Balakrishna, M. S.; Kaboudin, B. Tetrahedron Lett. 2001, 42, 1127. (k) Kaboudin, B. Chem. Lett. 2001, 880. (1) Sardarian, A. R.; Kaboudin, B. Tetrahedron Lett. 1997, 38, 2543. (m) Sardarian, A. R.; Kaboudin, B. Synth. Commun. 1997, 27, 543. (n) Kaboudin, B. Phosphorus Sulfur Silicon Relat. Elem. 2002, 177, 1749. 